\title{
Large eddy simulations of the HIFiRE scramjet using a compressible flamelet/progress variable approach
}

Amirreza Saghafian, Lee Shunn, David A. Philips, and Frank Ham

Cascade Technologies Inc., Palo Alto, CA, 94303, USA

Corresponding author: Lee Shunn

Mailing address:

Cascade Technologies, Inc.

2445 Faber Pl., Suite 100

Palo Alto, CA 94303-334

Fax: (650) 521-0679

Email: shunn@cascadetechnologies.com

Colloquium: 8. DETONATIONS, EXPLOSIONS and SUPERSONIC COMBUSTION

Total length: 6166 words (Method 2)

Color reproduction charges: The authors will not pay color reproduction charges. 


\title{
Large eddy simulations of the HIFiRE scramjet using a compressible flamelet/progress variable approach
}

\author{
Amirreza Saghafian, Lee Shunn, David A. Philips, and Frank Ham \\ Cascade Technologies Inc., Palo Alto, CA, 94303, USA
}

\begin{abstract}
In this study, large eddy simulations (LES) of supersonic combustion using a compressible flamelet-progress variable (CFPV) approach are performed for the HIFiRE scramjet at Mach 8 flight conditions. The LES results show good agreement with the ground test experimental measurements. The combustion model is based on an efficient flamelet-based approach, where a detailed chemistry mechanism is used in a pre-processing step. In the CFPV model, compressibility corrections are devised based on assumed functional forms of important thermo-chemical quantities. Specifically, the source term of the progress variable is rescaled with the local density and temperature in the LES, leading to improved predictions relative to traditional flamelet models. A modified equilibrium wall-model, capable of predicting the viscous heating, is used in the viscous near-wall region. Temperature near the wall increases significantly due to viscous heating, enhancing the reaction rate and heat-release. This is shown to be a crucial step for accurately predicting the pressure rise in the combustor.

Keywords: Supersonic Combustion, Turbulent Combustion, Compressible Flow, Scramjet, Large Eddy Simulation
\end{abstract}

\section{Introduction}

Scramjet engines have the potential to provide economical air-breathing propulsion at high Mach numbers for applications such as transportation, reusable launch vehicles, and hypersonic cruise missiles. Designing stable high-speed combustion systems remains a major challenge for scramjet propulsion, where fuel must be mixed, ignited, and burned to completion all 
within a few milliseconds. The large scale and extreme thermodynamic conditions in hypersonic flight vehicles make experiments costly and challenging [1]. Data are often limited to relatively sparse measurements of pressure, temperature, thrust, or combustion efficiency using conventional instrumentation. Validated numerical models are the most promising option to bridge the gap between sub-scale ground testing and full-scale flight-testing.

The vast majority of computational work in supersonic turbulent combustion has so far relied on simplified/reduced chemistry mechanisms and the explicit transport of the involved species [2]. Such approaches require the closure of the chemical source term in the species transport equations due to nonlinear interactions with the turbulent flow field. Although many types of closure models have been investigated $[3,4,5,6,7,8,9,10]$, research is ongoing to construct models that describe fundamental physics, reduce computational expense, and increase accuracy and robustness of simulation tools.

Flamelet models present an alternative to traditional finite rate chemistry approaches for CFD. The approach is based on the flamelet concept [11, 12], which assumes that the chemical time scales are shorter than the turbulent time scales, such that the flame can be approximated as an ensemble of laminar flamelets. The flamelet approach allows the computation of the chemistry to be performed independently of the flow simulation and stored in tabulated form as a function of a small number of scalars. During the CFD simulation, thermochemical quantities of interest are read and interpolated from the chemistry table, thus, dramatically decreasing the computational cost and allowing the use of complex chemical mechanisms.

In this study, a computationally efficient flamelet combustion model is developed for high-speed flows. Flamelet-based models that are currently used in low-Mach applications are extended and validated for hypersonic conditions. The target configuration is the HIFiRE Direct-Connect Rig experiments from the NASA Langley Arc-Heated Scramjet Test Facility [13].

\section{Mathematical model}

In this section, the filtered transport equations for LES of compressible reacting flows are presented, the combustion model based on the flamelet/progress variable approach is described, and the wall modeling approach is summarized. 


\subsection{Governing equations}

The Favre averaged or filtered variables (indicated by . and - respectively), $\bar{\rho}, \bar{\rho} \widetilde{u}_{i}, \bar{\rho} \widetilde{E}, \bar{\rho} \widetilde{Z}, \bar{\rho} \widetilde{Z^{\prime \prime 2}}$, and $\bar{\rho} \widetilde{C}$ are solved in the conservative form, where $\bar{\rho}$ is the density, $\widetilde{u}_{i}$ the components of the velocity vector, $\widetilde{E}$ the total energy (including the chemical energy), $\widetilde{Z}$ the mixture fraction, $\widetilde{Z^{\prime \prime 2}}$ the variance of the mixture fraction, and $\widetilde{C}$ a reaction progress variable [14].

The mixture composition depends directly on $\widetilde{Z}, \widetilde{Z^{\prime \prime 2}}$, and $\widetilde{C}$. In order to close this system, the pressure and the temperature need to be determined. This is achieved through using an equation of state and the energy of the mixture as explained in the following section. For the current study, the progress variable is defined as $C=Y_{\mathrm{H}_{2} \mathrm{O}}+Y_{\mathrm{H}_{2}}+Y_{\mathrm{CO}_{2}}+Y_{\mathrm{CO}}$.

\subsection{Combustion model}

The combustion model used in this study is based on the compressible flamelet/progress variable approach (CFPV) $[15,14]$. In the CFPV approach, flamelet equations are solved in a preprocessing step using detailed chemistry mechanisms (GRI 3.0 mechanism [16] in this study) and stored in a chemical table. Appropriate compressibility corrections are introduced [14] to decrease the size of the chemical table. The CFPV model has been extensively validated in a priori and a posteriori study using direct numerical simulation data [14]. In this section, the CFPV approach is briefly explained, and the compressibility corrections are summarized.

The CFPV approach is designed to account for the variations due to compressibility, while maintaining roughly the same computational efficiency as its low-Mach-number counterpart. In this approach, the thermochemical state of the compressible flow is approximated as a perturbation about nominal low-Mach-number number flamelet solutions. Appropriate expressions are derived based on the physical behavior of each variable, and are used as compressibility corrections to the nominal value which is interpolated from a low-Mach-number flamelet library.

Quantities of interest that are necessary to close the transport equations include the gas constant, specific heat ratio, temperature, pressure, molecular viscosity and thermal diffusivity. Corrections that account for the effects of compressibility on these quantities are described in detail in $[14,17]$.

The source term of the progress variable is very sensitive to perturbations in temperature and concentration. A rescaling for this term is applied to 
account for these perturbations. The source term in the progress variable transport equation is rescaled as

$$
\frac{\overline{\dot{\omega}}_{C}}{\overline{\dot{\omega}}_{C_{0}}}=\left(\frac{\bar{\rho}}{\bar{\rho}_{0}}\right)^{a_{\rho}} \exp \left[-T_{a}\left(\frac{1}{\widetilde{T}}-\frac{1}{\widetilde{T}_{0}}\right)\right],
$$

where $\overline{\dot{\omega}}_{C_{0}}$ is the tabulated source term computed at a background pressure $p_{0}$. This equation is the dimensionless ratio of the progress variable source term from the LES to its value from the chemistry table. It is used to adjust the tabulated production rate for consistency with the local temperature and density in the LES. The values of the baseline density $\bar{\rho}_{0}$, the density exponent $a_{\rho}$, and the activation temperature $T_{a}$ are computed in a preprocessing step to describe the dependency of the source term on the mixture temperature and pressure, and are then tabulated as a function of $\widetilde{Z}, \widetilde{Z^{\prime \prime 2}}$, and $\widetilde{C}$. The form of this equation is motivated by the Arrhenius behavior of elementary reactions, where density (to some order) appears in the species concentration terms. For hydrocarbon fuels, the value of $a_{\rho}$ is close to two, because the majority of reactions are bimolecular. The value of $T_{a}$, in general, depends on the chemical mechanism. For the conditions studied in this work, $T_{a}$ at stoichiometric conditions is approximately $15000 \mathrm{~K}$.

It should be noted that all of the variables with the " 0 " subscript are computed directly from the base-state flamelet data, while the other parameters, $a_{\rho}$ and $T_{a}$, are determined by local data fitting using flamelet solutions at perturbed conditions. All of the parameters are tabulated as functions of $\widetilde{Z}, \widetilde{Z^{\prime \prime 2}}$, and $\widetilde{C}$ in the chemistry table. The functional form of the compressibility corrections are general and can be used for different conditions and fuels (e.g., see [14] for hydrogen fuel). However, most of the compressibility coefficients are not universal (e.g., $T_{a}$ ), and are precomputed as functions of $\widetilde{Z}, \widetilde{Z^{\prime \prime 2}}$, and $\widetilde{C}$ for the chemistry and conditions of interest. For more details on the CFPV model along with validations of the above expressions refer to $[15,14]$.

\subsection{Wall modeling}

Wall-resolved LES of scramjets is not practical with the current computational resources [18], therefore, a wall model for the wall shear stress and heat flux is applied. The unstructured LES grid is defined all the way to the wall and is designed to resolve outer-layer scale motions of the boundary 
layer [19]. The wall model then solves the compressible equilibrium boundary layer equations for momentum and energy as described in [20, 21]. In the current work, the manner of exchanging data between the LES and wall model has been modified from Bodart and Larsson [20] to use an integral constraint based on the wall-adjacent LES cell. The following equations,

$$
\frac{1}{\delta} \int_{0}^{\delta} u_{\|} d \eta=\widetilde{u}, \quad \frac{1}{\delta} \int_{0}^{\delta} T d \eta=\widetilde{T},
$$

are solved iteratively at each wall-adjacent LES cell, ensuring that the average streamwise velocity and temperature from the wall model match the filtered values from the LES. The wall-shear stress computed by the wall-model is applied as a flux in the LES momentum equation. This integral approach allows for the wall-modeled viscous heating to be passed as a source term to the LES energy equation. In prior wall-modeling implementations, thermal feedback to the LES solution was limited to the wall heat flux and was simply zero in the adiabatic case.

\section{HIFiRE simulations}

Figure 1 shows the HIFiRE Flight 2 (HF2) scramjet [22] with relevant dimensions shown. At each streamwise fuel injection station shown in Fig. 1 , there are four injectors (spaced equally in the spanwise direction) on the body side (upper surface) and four injectors on the cowl side (lower surface). The primary injectors have a diameter of $3.175 \mathrm{~mm}$, and are canted at $15^{\circ}$ relative to the combustor wall. The secondary injectors are normal to the combustor wall with a diameter of $2.388 \mathrm{~mm}$. For more details on the HF2 geometry refer to [23].

The fuel is a JP-7 surrogate with molar composition of $64 \%$ ethylene and $36 \%$ methane. The total equivalence ratio is 1.0 , where $40 \%$ of the fuel by mass is injected from the primary injectors and the balance from the secondary injectors [23].

The experimental measurements [13] of the full-scale HIFiRE scramjet are conducted at NASA's Arc-Heated Scramjet Test Facility [24]. In this study, the experimental results corresponding to the highest Mach number are considered, where the Mach number at the isolator inlet is 3.46 (equivalent to the Mach 8 HF2 flight test). 


\subsection{Boundary conditions}

The LES is performed on one-quarter of the HF2 domain (including the cowl-side surface and one side wall), with symmetry boundary conditions applied at the mid-plane in both the spanwise and lateral directions. The use of symmetry boundary conditions reflects a compromise between computational costs and the need to provide adequate resolution in boundary layers, shear layers, and reaction zones. Unfortunately, the resolution requirements of the HF2 configuration made it infeasible to simulate the entire domain with the computational resources that were available. The symmetry boundary condition is obviously an approximation to the physical combustor and carries implications for the overall fidelity that can be achieved. Despite this, it is a reasonable concession given the cost constraints and has been used in other investigations $[25,26]$. Simulations of the full HF2 domain (i.e., no symmetry boundary condition) are planned as future work; however, results are not available for the current paper.

Specific inflow boundary conditions of the isolator, primary injectors, and secondary injectors are shown in Table 1. At the isolator inlet, synthetic turbulence is generated using a digital filtering approach [27, 28]. Profiles of the Reynolds stress tensor from the boundary layer DNS data of Wu and Moin [29] are rescaled and used as inputs. As the turbulent conditions inside the HIFiRE isolator are not well characterized [23, 22], the objective of this approach is simply to develop a fully turbulent boundary layer prior to the primary fuel injectors. This is similar to the approach taken in Brès et al. [30], where for practical design studies the synthetic turbulence inside a jet nozzle is prescribed in a manner to ensure realistic turbulence levels at the nozzle exit. Simulation of the full isolator provides a sufficiently long development length for the boundary layer to equilibrate before reaching dynamically important regions of the flow. All of the walls are assumed to be adiabatic due to the long duration of the experiment [13], and wall modeling is applied to all surfaces. Characteristic boundary conditions are applied at the outflow boundary.

\subsection{Numerical implementation}

The unstructured finite volume LES solver "Chris" developed at Cascade Technologies, Inc. [31] is employed in this study. Numerical stencils based on higher-order polynomial expansions are used to minimize numerical dissipation. The spatial discretization relies on a second-order hybrid central/ENO method, in which a shock sensor is used to identify the cells where the ENO 
scheme should be applied. An explicit third-order Runge-Kutta scheme is used for temporal integration. All sub-grid turbulent quantities are modeled using the dynamic procedure $[32,14]$.

A body-fitted, block-refined, unstructured mesh [31] is used for all of the simulations in this study. Several embedded zones of refinement are defined to provide sufficient resolution in regions where gradients are large, e.g., in boundary layers, fuel/air mixing layers, and the cavity. The simulations are performed using a coarse mesh with $21 \mathrm{M}$ cells, and a fine mesh with $96 \mathrm{M}$ cells to evaluate the grid independence of the solution. For the fine case, grid spacing in the wall normal direction near the primary injectors is approximately 100 wall units and is around $3 \%$ of the diameter of the primary injectors in the mixing layer of fuel and air. Grid spacing is approximately $35 \%$ larger in the coarse mesh. Although the coarse mesh includes the entire HF2 geometry, the fine mesh utilizes a shortened isolator in order to reduce the cost of the simulations. The impact of these decisions on the results are discussed below.

\subsection{Results}

Figure 2 shows the LES wall pressure distribution in the symmetry plane normal to the spanwise direction (centerline between the injectors). Experimental measurements on the body and cowl sides are also plotted for comparison. Statistics from LES are obtained by time-averaging instantaneous flow-fields after 10 flow-through times (when the LES results are statistically steady). A schematic of the HF2 domain is shown on the plot for reference.

Comparisons with the experimental measurements show relatively good agreement. Combustion heat-release causes a sharp pressure rise beginning just upstream of the primary injectors. The pressure reaches a plateau in the cavity, and rises again due to compression on the ramp wall of the cavity. Strong turbulent mixing produces a relatively homogeneous flow in the cavity, and nearly constant pressure in this region. The coarse LES under-predicts the pressure in the cavity by about $4.5 \%$, while the fine LES attains the correct pressure levels. The pressure drops suddenly as the supersonic flow expands at the end of the ramp wall. The pressure is under-predicted in the expansion region downstream of the secondary injectors on both the fine and coarse mesh; however, experimental uncertainty is uncharacteristically high in this region for reasons that are not explained in [13]. For example, note the large variations in the experimental measurements on the body and cowl 
sides around the secondary injectors. For this reason, the LES results are believed to be in the range of experimental uncertainties.

There are notable differences between the fine and coarse LES results in Fig. 2. The fine mesh outperforms the coarse grid in predicting trends and peak pressures in the cavity, ramp, and secondary injection regions. The coarse mesh, however, seems to better predict the initial pressure rise around the primary injectors. This discrepancy is most likely the result of the truncated isolator that was used in the fine grid simulations. The shorter development length resulted in jet/boundary-layer interactions that were sufficiently different from the coarse mesh to suppress mixing and combustion until the cavity. These differences will be clarified in future simulations.

It should be noted that the current combustion model uses a single mixture fraction that cannot fully represents the interactions between the primary and secondary fuel jets. Properly modeling this interaction could enhance the combustion around the secondary injectors, and increase the pressure in this region. The addition of a second mixture fraction and modeling of cross-dissipation effects in the flamelet equations are the subjects of ongoing research. Using a multi-stream model could potentially improve the results in the regions after the secondary injectors.

Figure 3 shows the mixture fraction contours and the pressure rise on the combustor walls (the isolator wall is excluded for clarity). The primary jets are confined near the wall by the relatively strong cross-flow. The secondary jets, which are injected normal to the cross-flow and have a higher momentum flux ratio, penetrate more deeply into the combustor interior. Figure 4 shows the iso-surface of the stoichiometric mixture fraction colored by the progress variable. Upstream of the cavity, the primary jets burn mostly in the low speed boundary layer, and the mixing region between the injectors. In fact, Figs. 3 and 4 indicate that the fuel streams merge before entering the cavity. Mixing is enhanced in this region as the boundary layer separates just prior to the cavity due to the strong adverse pressure gradient (see Fig. 2). This can be clearly seen in Fig. 5, where a small recirculation zone is visible between the primary injectors and the cavity step.

Contours of the progress variable near the primary injectors are plotted in Fig. 6a. The oxidizer stream in the mixing layer has low velocity and high temperature due to conversion of the kinetic energy to thermal energy. This provides favorable conditions for the mixture to ignite [14]. Auxiliary simulations were also performed to independently evaluate the effect of the source term correction outlined in Eq. 1, and the effects of the wall model. Figure 2 
compares the pressure distribution from the full CFPV model with a second LES where the source term correction was not applied. It is clear from the delayed pressure response in the case with no correction term that Eq. 1 is an indispensable step in the CFPV approach. Contours of the progress variable from these two cases are plotted in Figs. 6b and 6c, which shows that combustion cannot be sustained at the primary injectors without correcting the progress variable source term for compressibility. A third LES simulation was performed that included the source term correction but did not apply the wall model. Comparing Figs. 6a and 6c shows that the viscous heating provided by the wall model is equally important in stabilizing the flame at the primary injectors. The viscous heating in the isolator boundary layer transfers the kinetic energy of the flow into sensible energy. The corresponding increase in the fluid temperature enhances the reactions and stabilizes the flame near the primary injectors. Equation 1 provides a mechanism to couple these two phenomena in the CFPV model.

\section{Analysis and discussion}

Flamelet models assume that a turbulent reacting flow can be approximated by asymptotically-thin laminar flame structures embedded in a turbulent flow field [11]. Implicit in this model is the assumption that the chemical length and time scales of the flamelet are much smaller than the representative turbulent scales. Also, because flamelet manifolds are derived from collections of canonical flame structures, the target system must persist more or less within the prescribed mixing and turbulence regimes for the approach to be valid. In this section, the results from the HF2 simulations are evaluated in terms of assumptions common to the flamelet paradigm.

The CFPV model presented in Section 2.2 is based on non-premixed diffusion flamelets. It is instructive to compare characteristic flame structures from the compressible LES to the original incompressible flamelet solutions. Figure 7 shows a scatter plot of instantaneous temperature versus mixture fraction from the HF2 simulation. Data from two separate $z$-planes are shown - the dark symbols represent data from a slice along the centerline of the fuel injectors, and the lighter symbols show data from midway between the two jets. Laminar flamelet solutions depicting isenthalpic mixing $\left(\chi_{s t}=500 \mathrm{~s}^{-1}\right)$ and near equilibrium chemistry $\left(\chi_{s t}=1 \times 10^{-4} s^{-1}\right)$ are also presented for reference. 
Deviations from the incompressible flamelet manifold due to compressibility are immediately apparent in Fig. 7. Differences of over $1000 \mathrm{~K}$ relative to the baseline flamelets are observed in and around the reaction zone, with even larger temperature excursions near the lean boundary. These high temperatures arise from the shocked air flows in the isolator upstream of the primary mixers. Data sampled from the fuel jets (dark symbols) show temperature fluctuations around the baseline fuel inlet condition and a variety of reacting and intermediate states in the interior of the flamelet. Data sampled from between the fuel injectors (lighter symbols) suggest a more consistent transition to burning, as hot air is entrained with burning gases in this highly unsteady region. Profiles with similar flame structure and temperature range have been reported in previous RANS [33] and LES [25] studies of this configuration.

Although the global flame structure in Fig. 7 appears to have significant non-premixed character, more careful analyses are required to evaluate the suitabilty of the diffusion flamelet approach for the HF2 combustor. The Takeno Flame Index [34] is a useful indicator for identifying mixing regimes in reacting flows. It assumes that gradients in the fuel and oxidizer fields are aligned in premixed flames and anti-aligned in non-premixed flames

$$
G_{F O}=\frac{\nabla \tilde{Y}_{F} \cdot \nabla \tilde{Y}_{O}}{\left|\nabla \widetilde{Y}_{F}\right|\left|\nabla \widetilde{Y}_{O}\right|},
$$

so that non-premixed regimes are characterized by negative values of $G_{F O}$ and premixed regimes are identified by positive values of $G_{F O}$. Contours of $G_{F O}$ are shown in Fig. 5d. Non-premixed combustion modes are widespread in shear layers and the cavity; however, pockets of premixed combustion are peppered throughout the flow.

With the diverse range of flame states shown in Fig. $5 \mathrm{~d}$, it is interesting to consider which structures are contributing most strongly to the combustion. Figure 8 shows an iso-surface of progress variable reaction rate, a quantity that is closely correlated with heat release. The surfaces are colored according to the local value of the Flame Index. Non-premixed combustion is observed in the shear layer rollup at the windward base of the injectors, but there appears to be significant premixed zones in entrainment regions of the jetin-crossflow vortex systems.

A time-scale analysis is used to examine these reactive zones in more detail. The analysis is based on estimates of the local Damköhler number, 
$D a=\tau_{\text {flow }} / \tau_{\text {chem }}$. For non-premixed combustion, the scalar dissipation rate provides an inverse time scale for mixing. We use a model based on the equilibrium hypothesis proposed by De Bruyn Kops et al. [35]

$$
\frac{1}{\tau_{\text {flow }}}=\tilde{\chi}=\left(\frac{\widetilde{\nu}}{P e}+\frac{\nu_{t}}{S c_{t}}\right)|\nabla \widetilde{Z}|^{2},
$$

with the primary jet diameter as a representative length scale in the Peclet number. The computed dissipation rates are shown in Fig. 5c and are largely insensitive to the choice of length scale due to large contributions from the SGS term $\nu_{t} / S c_{t}$. The characteristic chemical time scale for the CFPV model is

$$
\tau_{\text {chem }}=\frac{\bar{\rho} \widetilde{C}}{\overline{\dot{\omega}}_{C}} .
$$

Contours of Damköhler number based on Eqs. 4 and 5 are shown in Fig. 5e.

The flamelet assumptions are valid for $D a \gg 1$, a criteria that can be loosely interpreted as $D a>10$. The regions shown in Fig. 8 are evaluated using this criteria in conjunction with the Flame Index to determine the suitability of the CFPV model for the HF2 case. Figure 9 shows the volume-weighted joint PDF of Damköhler number and Flame Index for the heat release regions shown in Fig. 8. The $D a=10$ line is represented by the horizontal dashed line and the non-premixed $\left(G_{O F}<0\right)$ and premixed $\left(G_{O F}>0\right)$ regions are divided by the vertical dashed line. A substantial fraction of the heat release region $(67 \%)$ lies in the upper left quadrant, where the flamelet assumptions are valid and diffusion flamelets properly characterize the combustion regime. A non-negligible fraction (26\%) of the heat release region falls in the upper right quadrant, indicating that time scale ratios are amenable to flamelet modeling but that the combustion model should consider premixed flame structures as a basis. The remaining $7 \%$ of the heat release region lies below the $D a=10$ line, suggesting that flamelet methods are unlikely to properly describe all of the important reaction phenomena under these conditions.

\section{Conclusions}

Large eddy simulations using the CFPV combustion model are reported for the HIFiRE scramjet at Mach 8 flight conditions. Results are compared with experimental measurements from the HIFiRE Direct-Connect Rig at 
NASA Langley. The CFPV model utilizes functional corrections to a nominal flamelet solution to account for compressibility, thus providing an efficient description of high-speed combustion with a low memory footprint. Turbulent wall modeling of the viscous near-wall region is used to accurately predict the wall shear stress and heat flux in the LES. The numerical results show good agreement with the experiment when both compressibility and wall effects are modeled. Neglecting either of these factors, however, leads to reduced combustion and poor prediction of the pressure trends observed in the experiment. Corrections to the progress variable source term are particularly important, as they provide strong coupling between the combustion chemistry and compressible phenomena such as shocks, expansions, and viscous heating. The present results also suggest that jet/boundarylayer interactions are important in sustaining proper mixing and combustion in the near field of the primary injectors. All of these effects must be considered to stabilize combustion in the proper location. A time-scale analysis showed that assumptions about flamelet time scales are valid throughout a large fraction of important heat release regions the HIFiRE combustor. However, analysis of the mixing regime suggested that premixed combustion plays a non-negligible role in this flow and should be considered in future simulations.

\section{Acknowledgments}

An award of computer time was provided by the Innovative and Novel Computational Impact on Theory and Experiment (INCITE) program. This research used resources of the Argonne Leadership Computing Facility at Argonne National Laboratory, which is supported by the Office of Science of the U.S. Department of Energy under contract DE-AC02-06CH11357. Ad-

ditional support was provided by the Air Force Research Laboratory STTR program under project number FA8650-12-C-2246.

\section{References}

[1] J. M. Tishkoff, J. P. Drummond, T. Edwards, A. S. Nejad, AIAA Paper 97-1017 (1997).

[2] K. Bray, Proc. Combust. Inst. 26 (1996) 1-26.

[3] R. Baurle, A. Hsu, H. Hassan, J. Propul. Power 11 (1995) 1132-1138. 
[4] D. Chakraborty, P. Paul, H. Mukunda, Combust. Flame 121 (2000) 195209.

[5] R. Baurle, S. Girimaji, Combust. Flame 134 (2003) 131-148.

[6] H. Möbus, P. Gerlinger, D. Brüggemann, Combust. Flame 132 (2003) $3-24$.

[7] F. Genin, B. Chernyavsky, S. Menon, AIAA Paper 2003-7035 (2003).

[8] S. Karl, K. Hannemann, A. Mack, J. Steelant, AIAA Paper 2008-2548 (2008).

[9] A. Ingenito, D. Cecere, E. Giacomazzi, Shock Waves 23 (2013) 481-494.

[10] M. Chapuis, E. Fedina, C. Fureby, K. Hannemann, S. Karl, J. Martinez Schramm, Proc. Combust. Inst. 34 (2013) 2101-2109.

[11] N. Peters, Turbulent combustion, Cambridge University Press, 2000.

[12] H. Pitsch, Annu. Rev. Fluid Mech. 38 (2006) 453-482.

[13] K. Cabell, N. Hass, A. Storch, M. Gruber, AIAA Paper 2011-2248 (2011).

[14] A. Saghafian, High-fidelity simulations and modeling of compressible reacting flows, Ph.D. thesis, Stanford University, 2014.

[15] A. Saghafian, V. E. Terrapon, F. Ham, H. Pitsch, AIAA Paper 20112267 (2011).

[16] G. Smith, D. Golden, M. Frenklach, N. Moriarty, B. Eiteneer, M. Goldenberg, C. Bowman, R. Hanson, S. Song, W. Gardiner, et al., see http://www. me. berkley. edu/gri_mech (1999).

[17] A. Saghafian, V. E. Terrapon, H. Pitsch, Combust. Flame (2014).

[18] J. Larsson, AIAA Paper 2012-4261 (2012).

[19] S. Kawai, J. Larsson, Phys. Fluids 24 (2012) 015105.

[20] J. Bodart, J. Larsson, in: Annual Research Briefs, Center for Turbulence Research, Stanford University, Stanford, CA, 2011. 
[21] G. I. Park, P. Moin, Phys. Fluids 26 (2014) 015108.

[22] K. R. Jackson, M. R. Gruber, S. Buccellato, AIAA Paper 2011-2202 (2011).

[23] A. M. Storch, M. Bynum, J. Liu, M. Gruber, AIAA Paper 2011-2249 (2011).

[24] R. W. Guy, R. C. Rogers, R. L. Puster, K. E. Rock, G. S. Diskin, AIAA Paper (2012) 96-1732.

[25] I. Bermejo-Moreno, J. Larsson, J. Bodart, R. Vicquelin, Annual Research Briefs, Center for Turbulence Research (2013) 3-19.

[26] J. Larsson, I. Bermejo-Moreno, J. Bodart, R. Vicquelin, Annual Research Briefs, Center for Turbulence Research (2012) 241-251.

[27] L. di Mare, M. Klein, W. P. Jones, J. Janicka, Phys. Fluids 18 (2006) 025107.

[28] E. Touber, N. Sandham, Theor. Comp. Fluid Dyn. 23 (2009) 79-107.

[29] X. Wu, P. Moin, Phys. Fluids 22 (2010) 085105.

[30] G. A. Brès, F. Ham, J. W. Nichols, S. K. Lele, AIAA Paper 2013-2142 (2013).

[31] G. A. Brès, J. W. Nichols, S. K. Lele, F. E. Ham, R. H. Schlinker, R. A. Reba, J. C. Simonich, AIAA Paper 2012-2213 (2012).

[32] P. Moin, K. Squires, W. Cabot, S. Lee, Phys. Fluids 3 (1991) 2746.

[33] J. R. Quinlan, J. C. McDaniel, T. G. Drozda, Guilhem, J. Oefelein, AIAA Paper 2014-3743 (2014).

[34] H. Yamashita, M. Shimada, T. Takeno, Proc. Combust. Inst. 26 (1996) $27-34$.

[35] S. M. De Bruyn Kops, J. J. Riley, G. Kosaly, A. W. Cook, Flow Turbul. Combust. 60 (1998) 105-122. 
Table 1: Inflow boundary condition parameters.

\begin{tabular}{lccc}
\hline Inflow boundary & Pressure $[\mathrm{kPa}]$ & Temperature $[\mathrm{K}]$ & Mach number \\
\hline Isolator & 40.3 & 736.2 & 3.46 \\
Primary injector & 105.7 & 293.3 & 1.0 \\
Secondary injetor & 290.7 & 301.1 & 1.0 \\
\hline
\end{tabular}




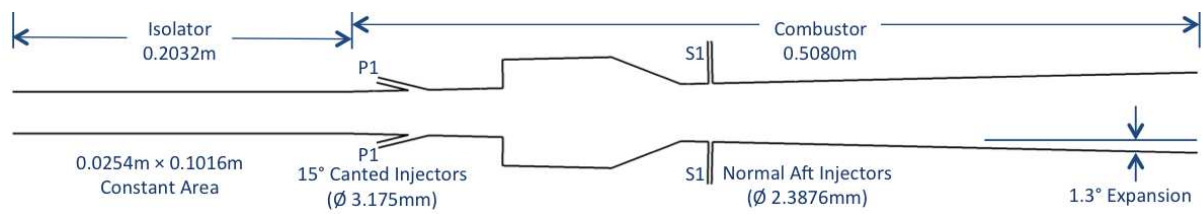

Figure 1: Schematic of HIFiRE 2 scramjet.

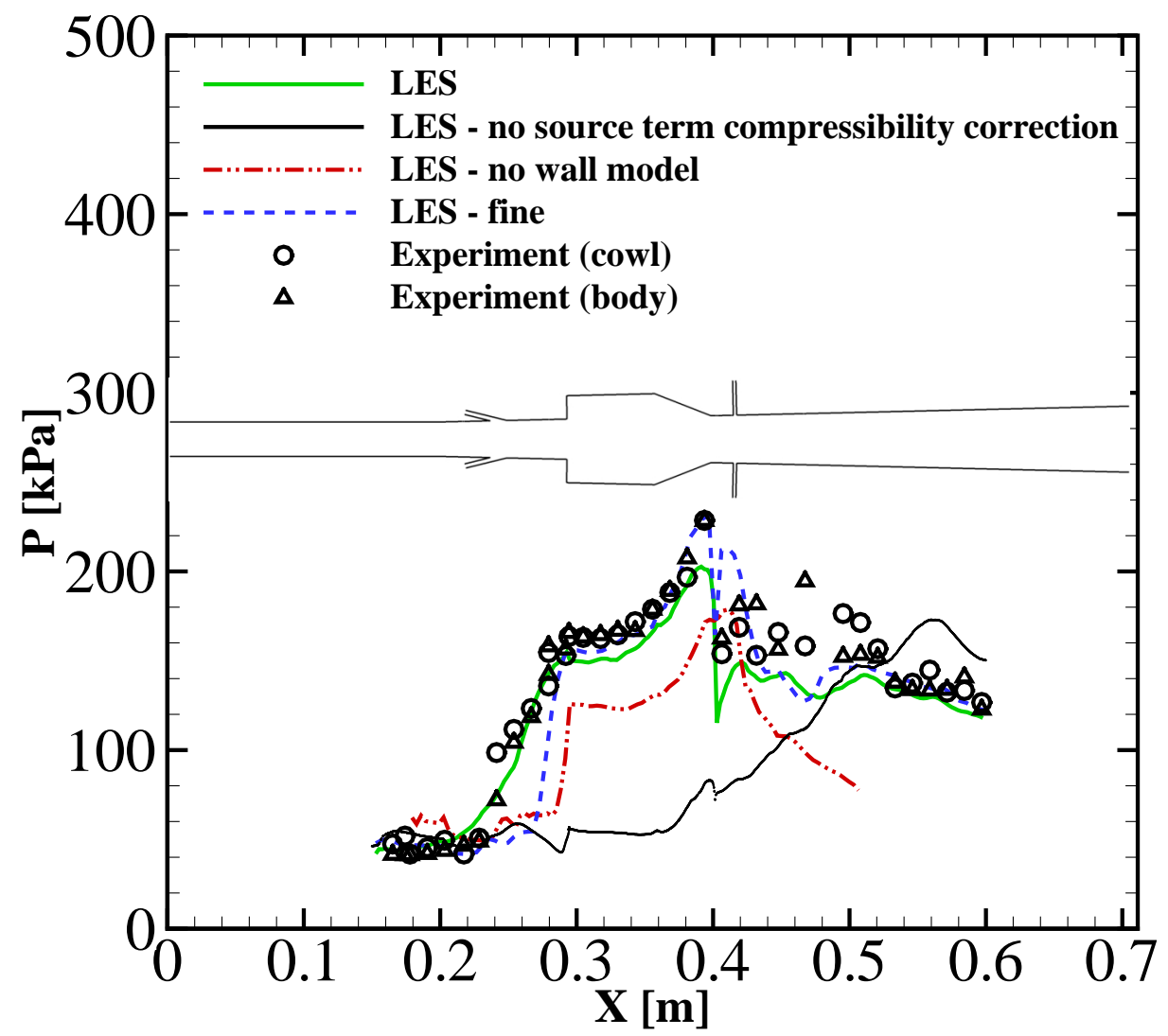

Figure 2: Wall pressure along the centerline between the injectors. LES results (solid lines) are compared with the experimental measurements (symbols). Black line: LES without progress variable source term corrections (i.e., Eq. 1 is deactivated); Red dot-dash line: LES results without wall model; Green line: LES on coarse mesh; Blue dotted line: LES on fine mesh. 


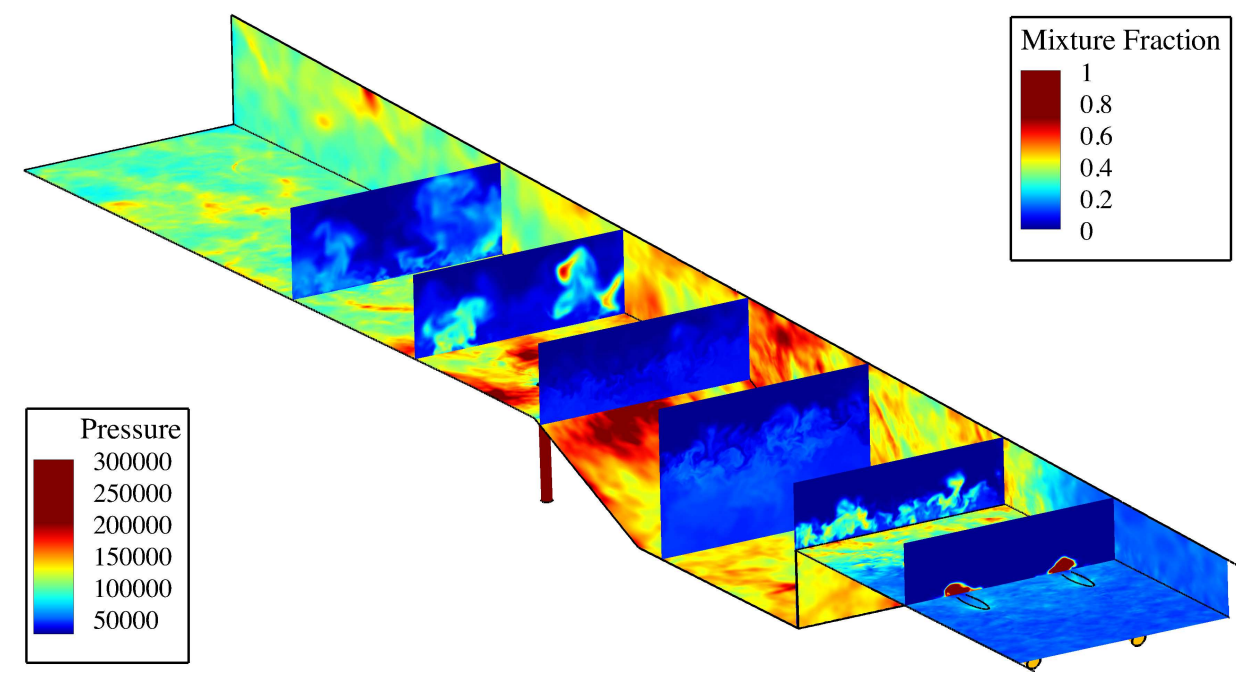

Figure 3: Contours of the mixture fraction on six slices normal to the streamwise direction at $x_{1}=0.25,0.3,0.35,0.4,0.45$, and $0.5 \mathrm{~m}$. Combustor wall is colored by pressure.

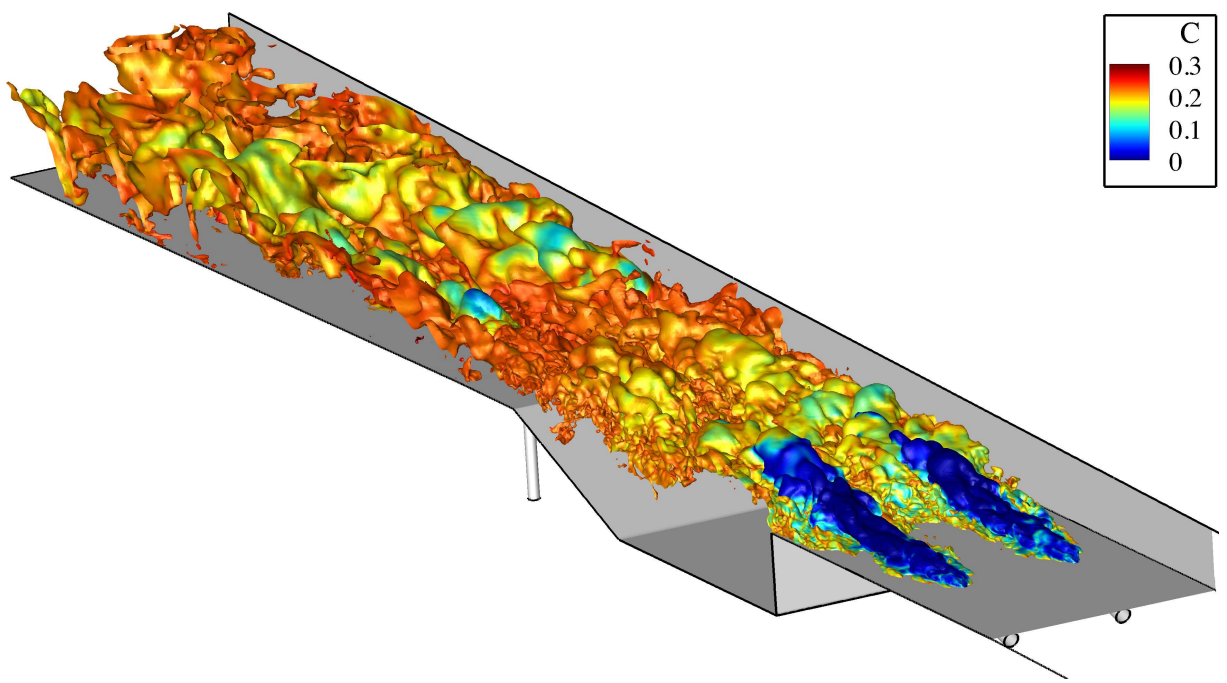

Figure 4: Iso-surface of the stoichiometric mixture fraction colored by the progress variable. 


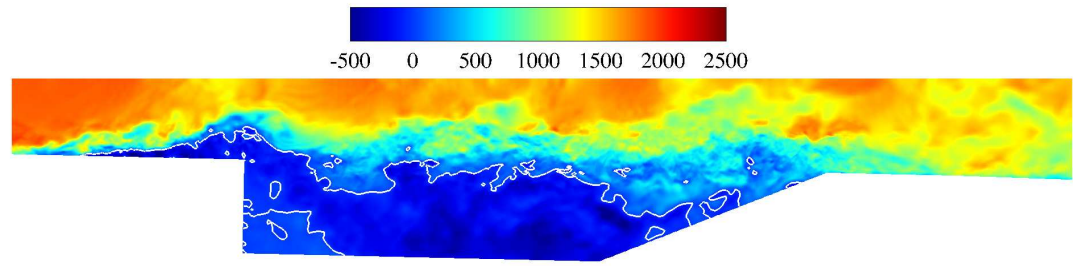

(a) Streamwise velocity (white line indicates the $u_{1}=0$ boundary)

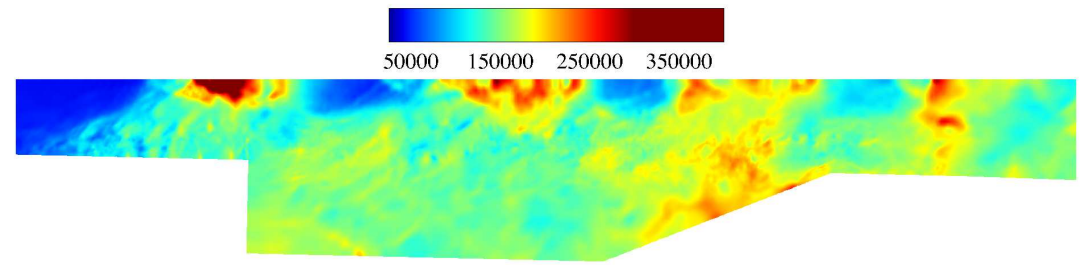

(b) Pressure

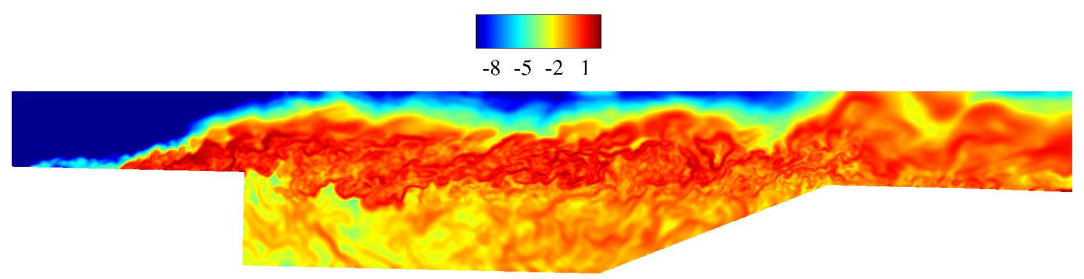

(c) Scalar dissipation rate, $\chi$ (logarithmic scale)

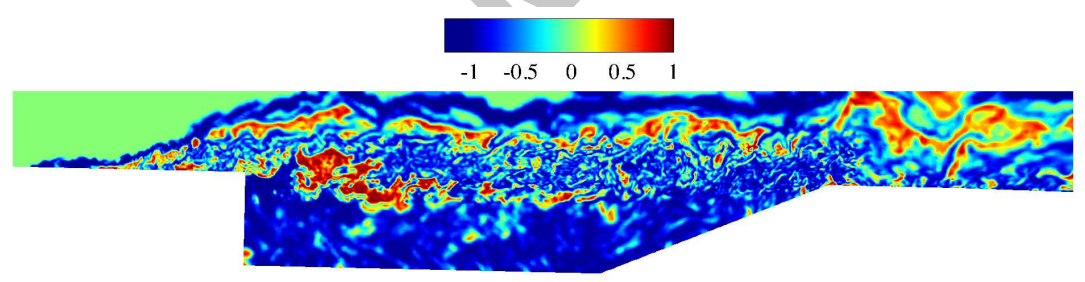

(d) Takeno Flame Index, $G_{F O}$

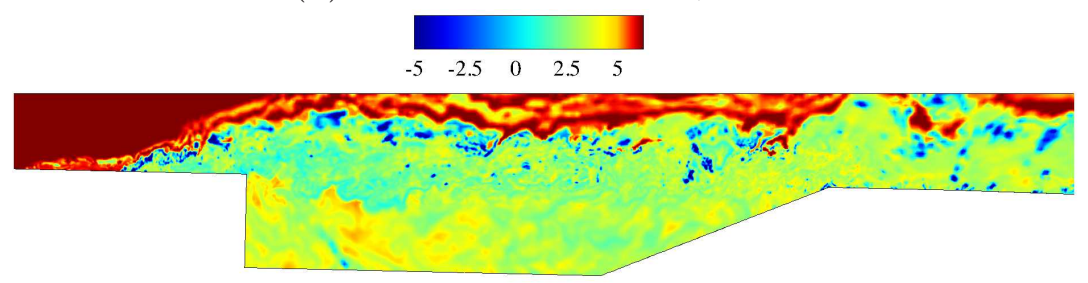

(e) Damköhler number (logarithmic scale)

Figure 5: Flow variables from LES of the HIFiRE scramjet. 


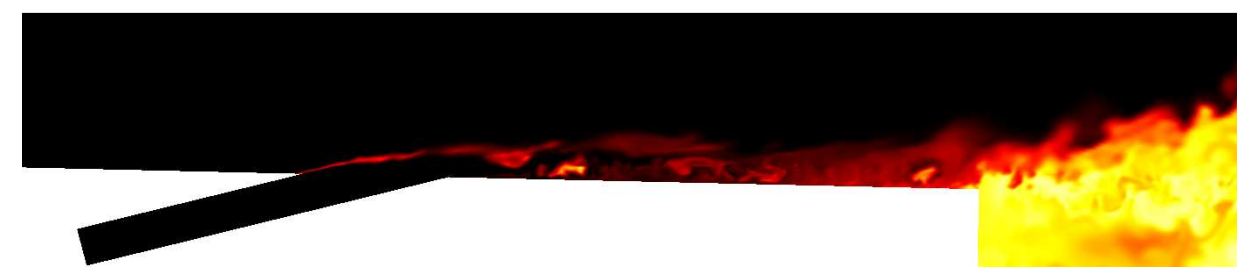

(a) with source term correction, with wall model

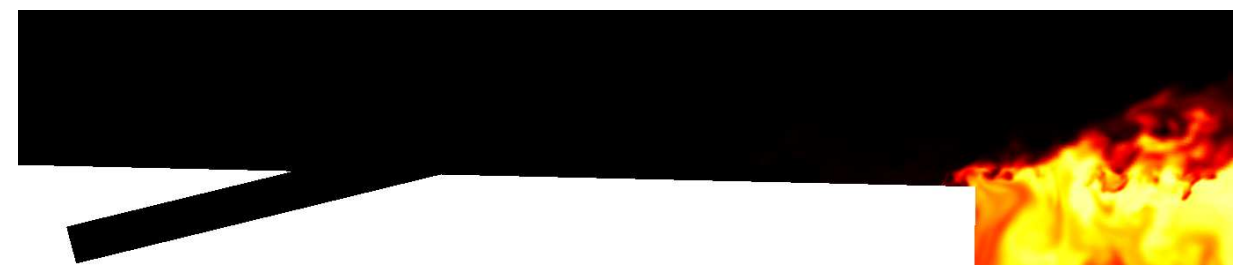

(b) without source term correction, with wall model

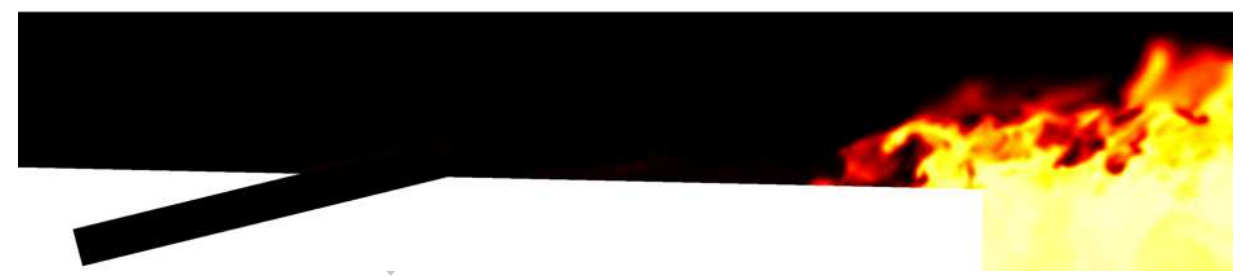

(c) with source term correction, without wall model

Figure 6: Contours of the progress variable from $C=0$ (black) to $C=0.3$ (white). 


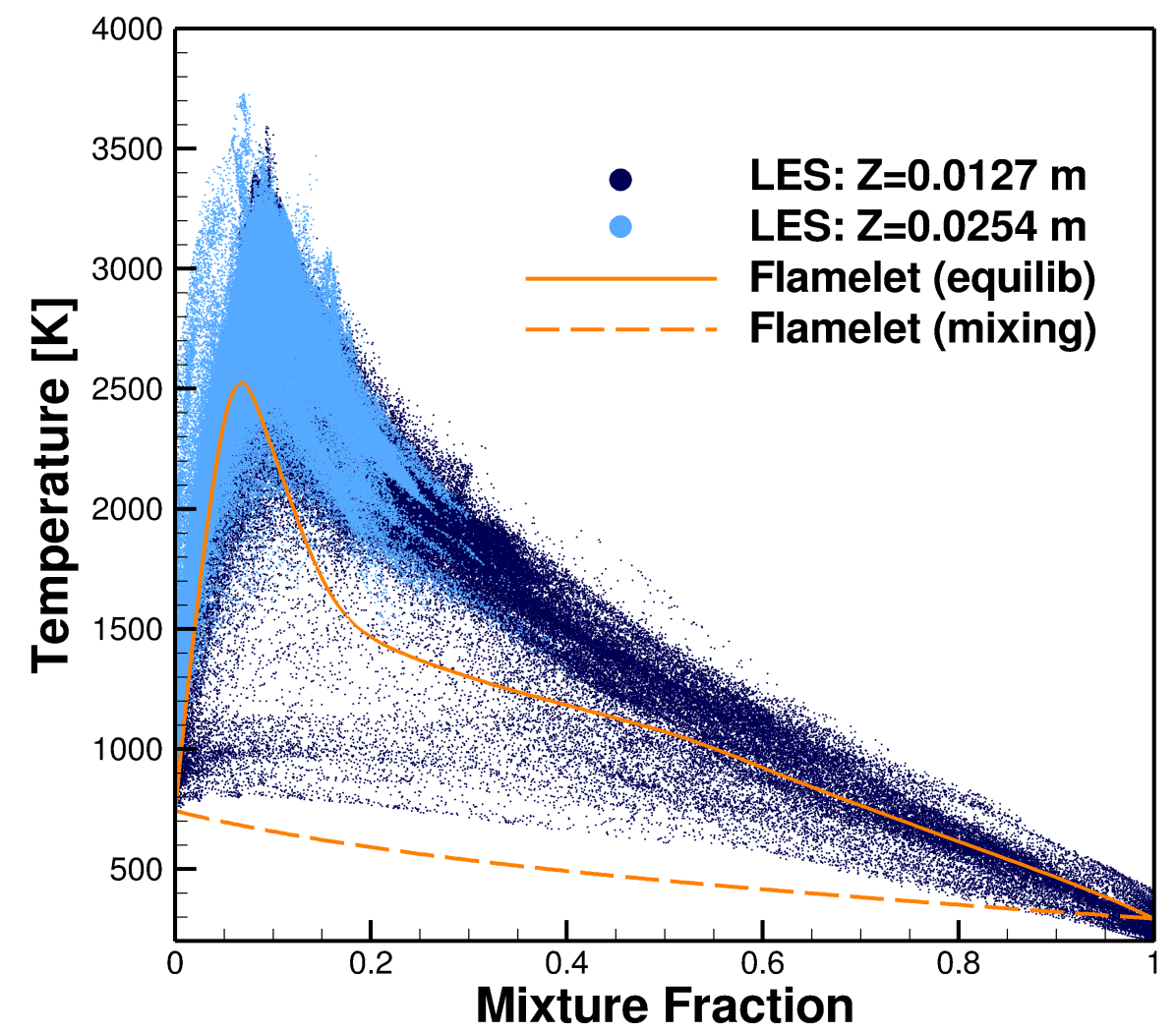

Figure 7: Scatter plot showing temperature $\widetilde{T}$ from the LES as a function of mixture fraction $\widetilde{Z}$. Representative solutions from the laminar flamelet library are shown for comparison. 


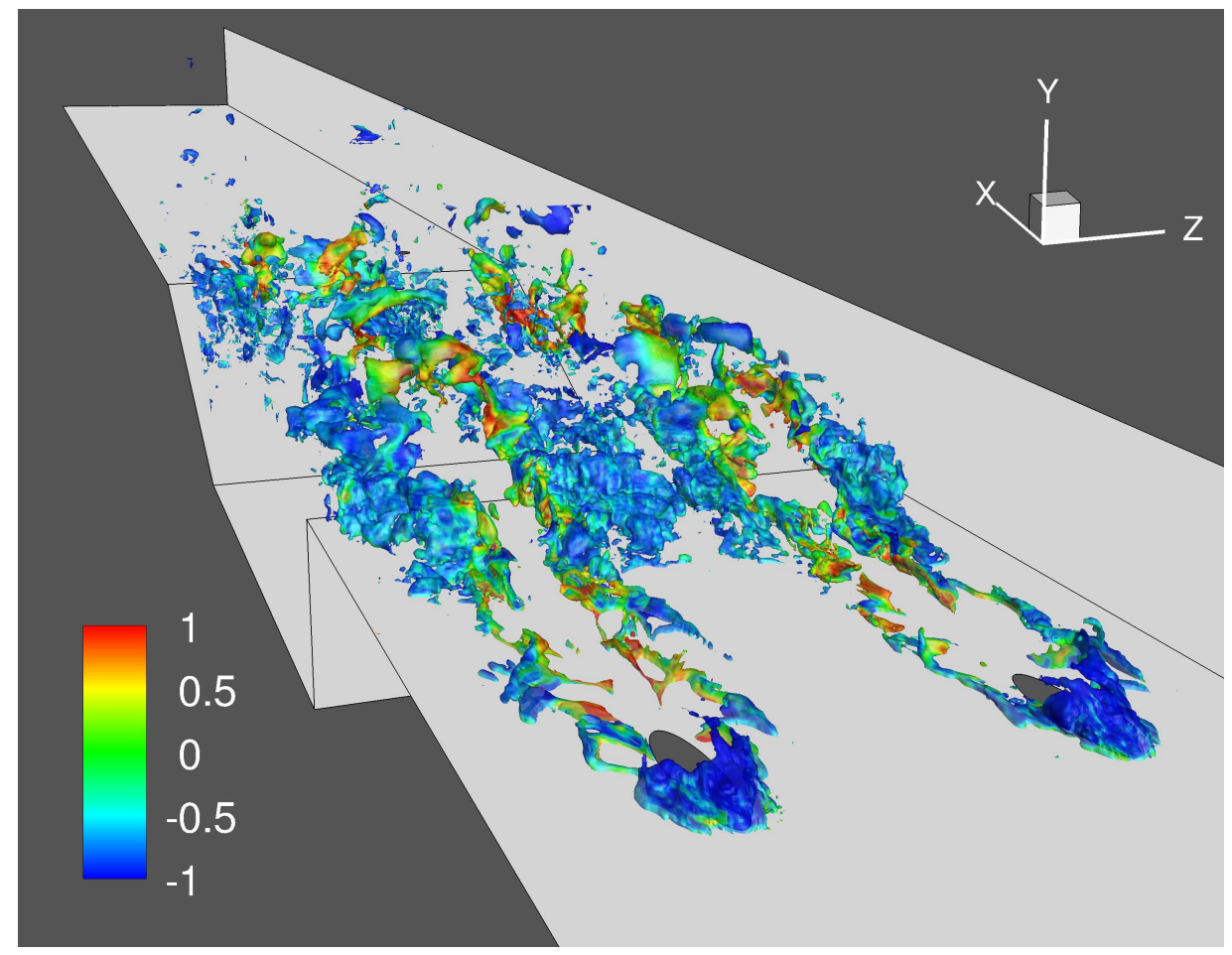

Figure 8: Iso-surfaces showing regions of intense reaction and heat release, colored by values of the Flame Index, $G_{F O}$. 


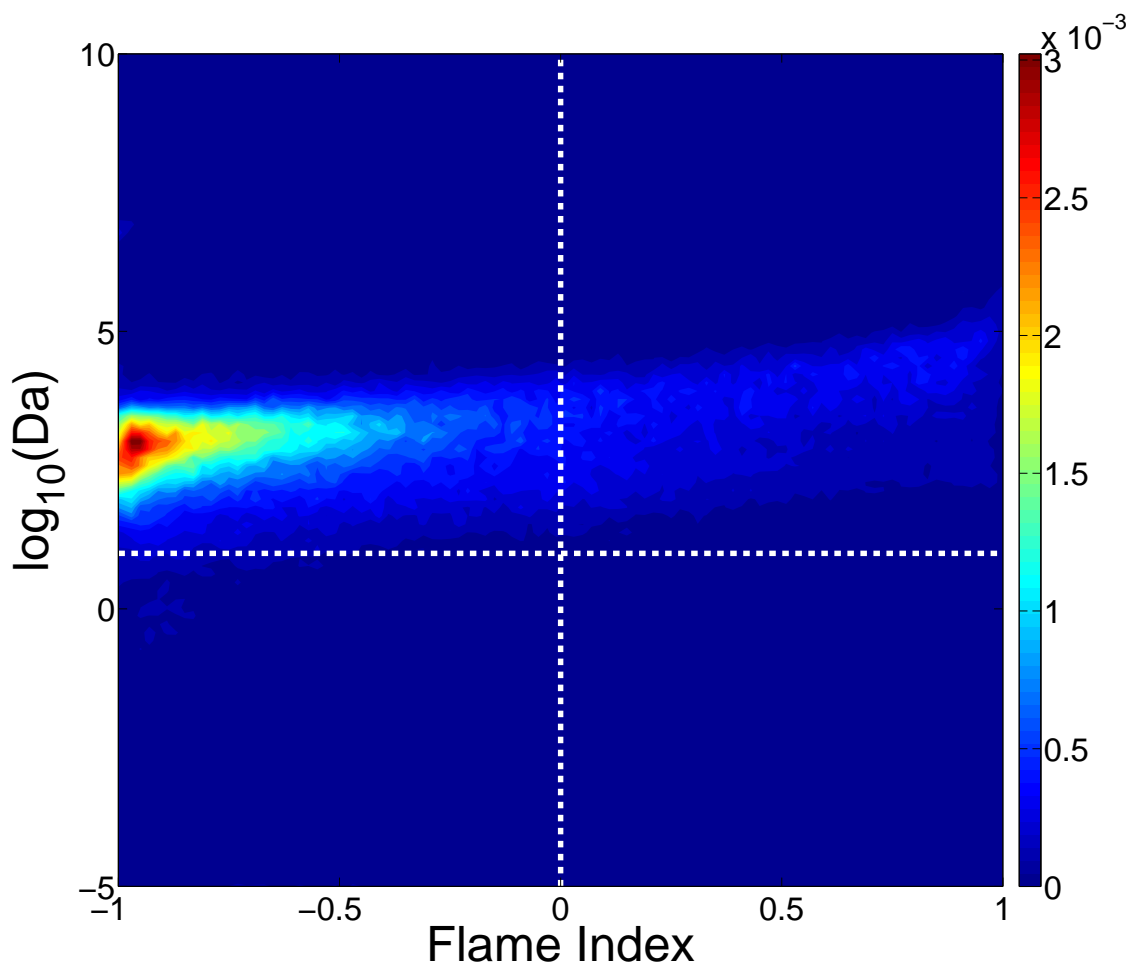

Figure 9: Volume-weighted joint PDF of Damköhler number and Flame Index for the heat release regions shown in Figure 8. 


\section{List of Figures}

1 Schematic of HIFiRE 2 scramjet. . . . . . . . . . . . . 16

2 Wall pressure along the centerline between the injectors. LES results (solid lines) are compared with the experimental measurements (symbols). Black line: LES without progress variable source term corrections (i.e., Eq. 1 is deactivated); Red dot-dash line: LES results without wall model; Green line: LES on coarse mesh; Blue dotted line: LES on fine mesh. . . . 16

3 Contours of the mixture fraction on six slices normal to the streamwise direction at $x_{1}=0.25,0.3,0.35,0.4,0.45$, and $0.5 \mathrm{~m}$. Combustor wall is colored by pressure. . . . . . . . 17

4 Iso-surface of the stoichiometric mixture fraction colored by the progress variable. . . . . . . . . . . . . . . 17

5 Flow variables from LES of the HIFiRE scramjet. . . . . . . . 18

6 Contours of the progress variable from $C=0$ (black) to $C=$ 0.3 (white). . . . . . . . . . . . . . . . 19

$7 \quad$ Scatter plot showing temperature $\widetilde{T}$ from the LES as a function of mixture fraction $\widetilde{Z}$. Representative solutions from the laminar flamelet library are shown for comparison. . . . . . . . 20

8 Iso-surfaces showing regions of intense reaction and heat release, colored by values of the Flame Index, $G_{F O}$. . . . . . . 21

9 Volume-weighted joint PDF of Damköhler number and Flame Index for the heat release regions shown in Figure 8. . . . . . . 22 